\title{
Periodontal diseases: is it possible to prevent them? A populational and individual approach
}

\author{
Luís Otávio Miranda COTA ${ }^{(a)}$ \\ Cristina Cunha VILLAR ${ }^{(b)}$ \\ Mario Vianna VETTORE(c) ID \\ Julya Ribeiro CAMPOS(a) \\ Guilherme Castro Lima Silva do \\ AMARAL (b) iD \\ José Roberto CORTELLI(d) iD \\ Sheila Cavalca CORTELLI(d) iD \\ (a) Universidade Federal de Minas Gerais - \\ UFMG, School of Dentistry, Department \\ of Dental Clinics, Oral Surgery and Oral \\ Pathology, Belo Horizonte, MG, Brazil. \\ (b) Universidade de São Paulo - USP, School of \\ Dentistry, Department of Stomatology, São \\ Paulo, SP, Brazil. \\ (c) University of Agder - UiA, Faculty of \\ Health and Sport Sciences, Department \\ of Health and Nursing Sciences, \\ Kristiansand, Norway. \\ (d) Universidade de Taubaté - Unitau, \\ Department of Dentistry, Taubaté, SP, Brazil.
}

Declaration of Interests: The authors certify that they have no commercial or associative interest that represents a conflict of interest in connection with the manuscript.

Corresponding Author: Luís Otávio Miranda Cota luiscota@ufmg.br

https://doi.org/10.1590/1807-3107bor-2021.vol35.0098

Submitted: March 12, 2021

Accepted for publication: March 31, 2021

Last revision: April 15, 2021

\begin{abstract}
Periodontal diseases are infectious inflammatory chronic diseases of a multifactorial nature. They are primarily caused by dysbiotic ecological changes in dental biofilm, which may be influenced by risk factors. The prevention of periodontal diseases may involve different strategies focused on reducing distal, intermediate, and proximal risk factors at both the population and individual levels. Effective prevention depends on interdisciplinary and common risk factor approaches. Also, patient-centered preventive models are more effective than professional-centered models in the management of periodontal diseases. Regular and periodic control of dental biofilm is an essential measure for the different levels of prevention of periodontal diseases. The effectiveness of periodontal disease prevention largely depends on positive modifications of behavior, knowledge, health literacy, patient empowerment, motivation, and compliance.
\end{abstract}

Keywords: Health Education; Periodontal Diseases; Public Health; Risk Factors.

\section{Introduction}

Periodontal diseases are microbially-associated, host-mediated inflammatory chronic diseases of a multifactorial nature. Biofilm accumulation and dysbiotic ecological changes are the primary causes of periodontal diseases, which may be influenced by risk factors leading to disease initiation and progression. ${ }^{1}$ Prevention of periodontal diseases may involve different strategies focused on tackling modifiable risk factors at both the population and individual levels within the causal chain throughout the disease process (Figure 1). Primary prevention refers to a preclinical stage with the aim of preventing the onset of the disease by reducing the risk factors of biofilm development and gingival inflammation. Secondary prevention concerns the proper management of the disease at an early stage based on timely diagnosis and prompt treatment to regain periodontal health. Tertiary prevention applies to established and irreversible disease in order to restrain the sequelae, restore functions, and prevent the recurrence of the disease. ${ }^{2}$

Effective prevention of periodontal diseases may be achieved through targeted and high-risk approaches aiming to reduce distal, intermediate, and proximal risk factors (Figure 1). The control of the periodontal causal chain is 
the cornerstone of success in prevention and periodontal therapy. ${ }^{3}$ Whole-population approaches focus on environmental conditions as the major determinants of the diseases to increase overall population health by shifting the distribution of population risk exposure toward a lower mean. ${ }^{4}$ Targeted approaches are directed at population groups who are at higher risk of periodontal disease (e.g. diabetic patients) through behavioral changes. High-risk approaches represent the traditional biomedical approach where susceptible individuals are identified and treated. ${ }^{4}$ Trends in patient care are shifting from the traditional biomedical model to comprehensive patient-centered approaches, ${ }^{2}$ whereby the individual is the central point in healthcare and the socioenvironmental context of that individual is considered.

The present manuscript addresses the prevention of periodontal diseases with focus on high-risk approaches, including oral hygiene instructions and mechanical or chemical biofilm control. Other preventive strategies that have been receiving attention recently are also included, as they have started changing the way clinicians manage the disease. They include complex educational programs involving psychological and behavioral changes, patient empowerment and self-management, and acquisition of therapeutic skills. ${ }^{2}$

\section{Mechanical biofilm removal}

Regular and periodic control of dental biofilm is an essential measure for the prevention and treatment of plaque-induced periodontal diseases. Management of gingivitis is the primary preventive determinant of periodontitis onset and the secondary preventive characteristic for periodontitis recurrence. ${ }^{5,6}$ Compelling evidence has shown that bleeding sites have $70 \%$ more attachment loss than non-inflamed sites. ${ }^{6}$ Effective daily tooth brushing is effective in reducing plaque from tooth surfaces. Accordingly, evidence from systematic reviews indicates that a single episode of manual tooth brushing reduces dental plaque by $42 \%$ on average, and greater benefits can be achieved with powered toothbrushes. ${ }^{7}$

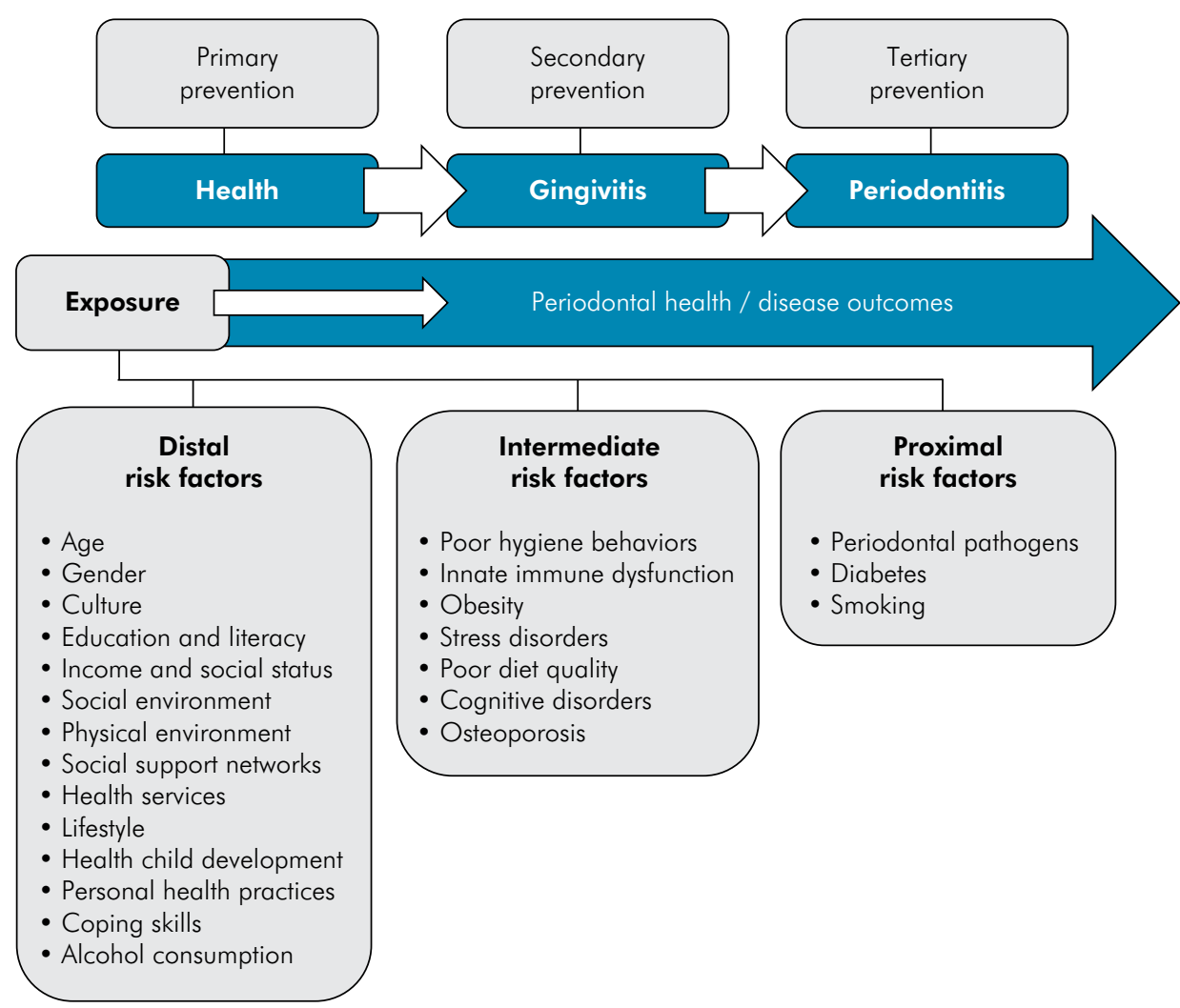

Figure 1. Periodontal risk factors. Adapted from Bouchard et al. ${ }^{3}$ 
Likewise, evidence suggests that the use of powered toothbrushes is more effective in the reduction of gingival inflammation than manual toothbrushes. Also, oscillating-rotating toothbrushes seem to produce better clinical outcomes. ${ }^{7}$ However, the importance of the reductions of dental plaque and gingivitis for the prevention of periodontitis is questionable, since no study has directly evaluated the long-term effects of dental plaque and gingivitis control in the stability of periodontal health.

Current evidence of the use of interdental plaque control indicates that the use of dental floss in combination with tooth brushing does not provide further benefits for dental plaque removal than tooth brushing alone, but may result in lower levels of gingival inflammation. However, patients with a history of periodontitis should use interdental brushes for interproximal plaque removal. The combined use of interdental brushes and tooth brushing promotes greater reductions in dental plaque and gingival inflammation as compared to tooth brushing alone or when associated with dental floss. ${ }^{8}$

Nevertheless, optimal levels of oral hygiene may not be achieved without prior removal of pre-existing heavy deposits. Therefore, professional mechanical plaque removal (PMPR) is frequently done with this purpose. Although there is a lack of robust data to enable definitive conclusions about the effects of PMPR on the primary prevention of periodontitis, low-to-moderate evidence indicates that PMPR, particularly when combined with oral hygiene instructions $(\mathrm{OHI})$, can result in greater reductions of plaque and gingival inflammation in patients with gingivitis. ${ }^{9}$ Existing knowledge also suggests that the magnitude of these effects is positively correlated with the frequency of PMPR. However, PMPR may not result in additional benefits for plaque and gingivitis control over those achieved by repeated $\mathrm{OHI}$ only in these patients. ${ }^{9}$ The direct effect of PMPR on the secondary prevention of periodontitis has not been clarified yet, since it is usually part of a comprehensive periodontal maintenance program.

\section{Chemical biofilm control}

The adjunctive use of antimicrobial agents to control dental plaque is indicated for patients with difficulties in maintaining appropriate plaque control through mechanical methods or reaching interproximal areas. In this context, dentifrices and mouthrinses with antimicrobial agents are efficient to help prevent gingivitis and produce better clinical outcomes than what can be accomplished with self-performed mechanical plaque control only. Overall, oral rinses with anti-plaque agents can reduce gingivitis by approximately $23 \%$, while tooth brushing with toothpaste reduces gingivitis by $21 \%{ }^{10}$ Even though systematic reviews suggest that mouthrinses consistently promote greater reductions in gingival inflammation than dentifrices, their real-life efficacy might be limited because patients would have to incorporate a new habit into their daily routine. Since dentifrices are commonly used by the general population, they might be considered more suitable to achieve sustainable clinical gains than mouthwashes. ${ }^{10}$

In general, most antimicrobial agents added to dentifrices (e.g. zinc citrate, stannous fluoride) generate similar and significant anti-plaque and gingivitis responses in patients with gingivitis. ${ }^{11}$ However, other agents, such as chlorhexidine (CHX) (0.1\%), can promote greater reductions in dental plaque and gingivitis. The possible benefits of dentifrices containing antimicrobials for the periodontal status of patients successfully treated remain unknown due to the scarcity of evidence. Nonetheless, available data suggest that the combined use of dentifrice and mouthrinse containing amine fluoride $(\mathrm{AmF})$ and stannous fluoride $(\mathrm{SnF} 2)$ reduces dental plaque levels by nearly $10 \%$. No further effects on other clinical parameters in patients undergoing periodontal maintenance have been observed..$^{10}$

There is sound evidence that the adjunctive use of CHX mouthrinse in patients with gingivitis can reduce dental plaque and gingival inflammation by $33 \%$ and $26 \%$, respectively (Table). However, the long-term use of $\mathrm{CHX}$ mouthrinse is limited because of side effects like tooth staining and alterations in taste. For long-term use, essential oils (EO) and cetylpyridinium chloride (CPC) are considered better therapeutic options, since both are effective in reducing dental plaque by $27 \%$ and $15 \%$ and gingivitis by $18 \%$ and $13 \%$, respectively. ${ }^{10,11}$ Notably, current evidence suggests that $\mathrm{EO}$ is a reliable alternative 
to $\mathrm{CHX}$ in the management of gingivitis in the long term. ${ }^{12}$ The long-term use of EO results in a higher percentage of sites with no gingivitis in comparison with mechanical control alone. ${ }^{13}$

The long-term use of CHX mouthrinse for secondary prevention has been shown to promote considerable reductions in dental plaque and gingivitis by approximately $20 \%,{ }^{14}$ but there is no data on the effectiveness of $\mathrm{CHX}$ mouthrinse to prevent periodontal disease recurrence. Additional studies are needed to investigate the effect of $\mathrm{EO}$ and CPC-based mouthrinses on secondary prevention.

\section{Common risk factor approach}

The common risk factor approach (CRFA) proposes an integrated strategy by addressing common risks to chronic conditions, including diabetes, cardiovascular diseases, cancers, and periodontal diseases, within a wider socioenvironmental context. ${ }^{15}$ The central concept underlying CRFA is that controlling a relatively small number of risk factors would positively impact on a considerable number of diseases (Figure 2). In addition, CRFA focuses on oriented

Table. Percentage of biofilm and gingivitis reduction according to antimicrobial agents.

\begin{tabular}{lcc}
\hline \multirow{2}{*}{ Antimicrobial agents } & \multicolumn{2}{c}{$\begin{array}{c}\text { Percentage of biofilm and } \\
\text { gingivitis reductions }\end{array}$} \\
\cline { 2 - 3 } & $\begin{array}{c}\text { Plaque index } \\
\text { Chlorhexidine (CHX) }\end{array}$ & $\begin{array}{c}\text { Gingival index } \\
\text { Essential oils (EO) }\end{array}$ \\
Cetylpyridinium chloride (CPC) & $27 \%$ & $26 \%$ \\
\hline
\end{tabular}

Based on Serrano et al. ${ }^{10}$ and Figuero et al. ${ }^{10}$ health policies for the whole population and for groups at risk rather than on disease-specific at-risk groups. Behavioral risk factors, such as smoking, alcohol consumption, unhealthy diet, and physical inactivity are frequently clustered in groups of people with greater vulnerability to chronic diseases. Health attitudes and individual behaviors are also influenced by the complex interactions between contextual socioeconomic, political, and cultural factors. ${ }^{2,16}$

Taking integrated action to reduce diseases of multifactorial causation by tackling several risk factors related to one or more diseases results in low cost, efficient and effective public health interventions. The use of CRFA to prevent and reduce periodontal diseases must be implemented through comprehensive health promotion strategies. The WHO has advocated a range of global public health actions to reduce tobacco consumption, such as those supporting the enforcement of bans on tobacco advertising and smoke-free environments. ${ }^{17}$ These actions serve as an example of effective public health strategies related to CRFA with the potential to reduce periodontal disease and other chronic diseases. The bi-directional relationship between periodontitis and diabetes is striking, and preventive and management strategies of both diseases are similar. Indeed, both conditions are of multifactorial etiology, share similar risk factors, require a patient-centered approach and therapeutic education, and their control relies on self-management and monitoring of the condition. ${ }^{14}$

Periodontal prevention depends on interdisciplinary approaches with other medical and dental specialties.

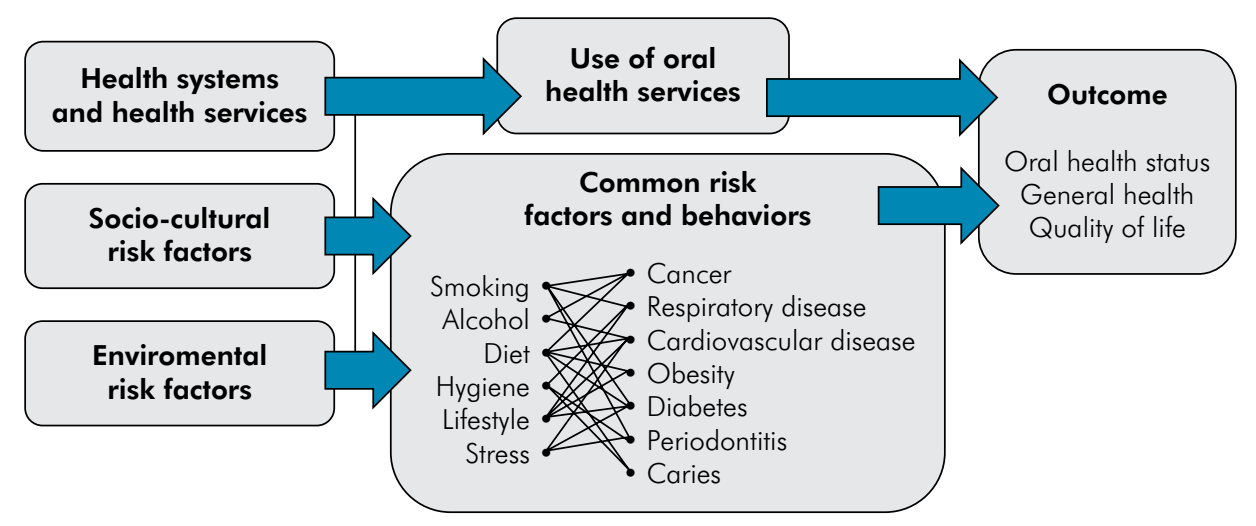

Figure 2. Risk factor approach in promotion of oral health. Adapted from Sheiham and Watt ${ }^{15}$ and $\mathrm{WHO} .^{16}$ 
Dentists tend to not participate in healthy lifestyle programs and there is a lack of knowledge about periodontal diseases among health professionals. There are several preventive programs to reduce chronic diseases in different countries, but oral health is rarely included in them. Hence, a shift towards CRFA to reduce the most common oral diseases, including periodontal disease, is needed in government preventive programs and strategies, with the participation and commitment of oral health professionals. ${ }^{18}$

\section{Socioeconomic position}

Socioeconomic position (SEP) indicates the social and economic aspects that influence what position individuals or groups hold within the structure of a society. SEP encompasses several exposures, resources, and susceptibilities that may influence health through relations of power and access to goods and services. Lower SEP is a well-identified risk indicator for periodontal diseases at contextual and individual levels. ${ }^{19,20}$ The use of SEP is essential to examine the role of SEP in health. Yet, distinct SEP indicators that are different in nature have been used with the same meaning in health research, such as social class, social stratification, social or socioeconomic status. Thus, evidence about this topic is unclear and questionable due to the different theoretical bases of SEP measures. Moreover, most studies addressing the social epidemiology of health were conducted in developed countries. Therefore, research outputs about this topic are limited for low- and middle-income countries. SEP indicators change throughout an individual's life (Figure 3) and should be chosen according to the objective of the study and the health outcome. ${ }^{21}$ The importance of theoretically-grounded SEP measures is crucial in epidemiological studies where SEP is the exposure of interest or a confounder.

There is an inverse relationship between SEP and periodontitis regardless of the measure of periodontitis. ${ }^{22}$ SEP indicators are frequently represented by a) Education; b) Housing tenure, conditions, and amenities; c) Income; d) Occupation; e) Proxy indicators; f) Wealth; g) Working life indicators and exclusion from the labor market; h) Composite indicators; i) Indices of deprivation, and j) Life course socioeconomic position. ${ }^{21}$ Particularly, education and income are strongly linked to health at both individual and population levels. To further elucidate how education is related to health, a conceptual framework had been proposed at the Organization for Economic Co-operation and Development (OECD) symposium, ${ }^{23}$ taking into consideration a wide range of concepts and interpretations (Figure 4).

\section{Health literacy}

Education is a common SEP indicator in periodontal research, assessed as a continuous (years of completed education) or categorical variable (e.g. complete high school). There is a dose-response relationship between education and periodontitis. ${ }^{22} \mathrm{~A}$ comprehensive theoretical model evaluating how education shapes health literacy was proposed (Figure 5). Attempts to encourage knowledge transfer from healthcare professionals to patients can be ineffective if the patients have low formal education levels. However, as healthcare moves toward a patient-centered approach with shared responsibilities, health education becomes even more relevant. Currently, patients with low health literacy skills and difficulties with reading, writing, and numeracy face tremendous limitations to assimilate healthcare information through electronic technology. ${ }^{24}$

The term health literacy was introduced in the 1970s and according to the World Health Organization refers to "The cognitive and social skills which determine the motivation and ability of individuals to gain access to understand and use information in ways which promote and maintain good health". ${ }^{25}$ The importance of health literacy increased throughout the last decades. More recently, the importance of moving from an individual-centered approach to a new one where demands of health systems interact with individuals' skills has been recognized. ${ }^{26}$

Health literacy is limited to an individual's capacities, while population health literacy is extended outside these borders. ${ }^{25}$ According to individual skills, health literacy can be classified in groups that range from basic skills (e.g. those related to daily life situations, such as reading and writing) to more advanced cognitive skills. If health literacy skills enhance autonomy and empowerment, health-related decision-making is also improved. Therefore, 

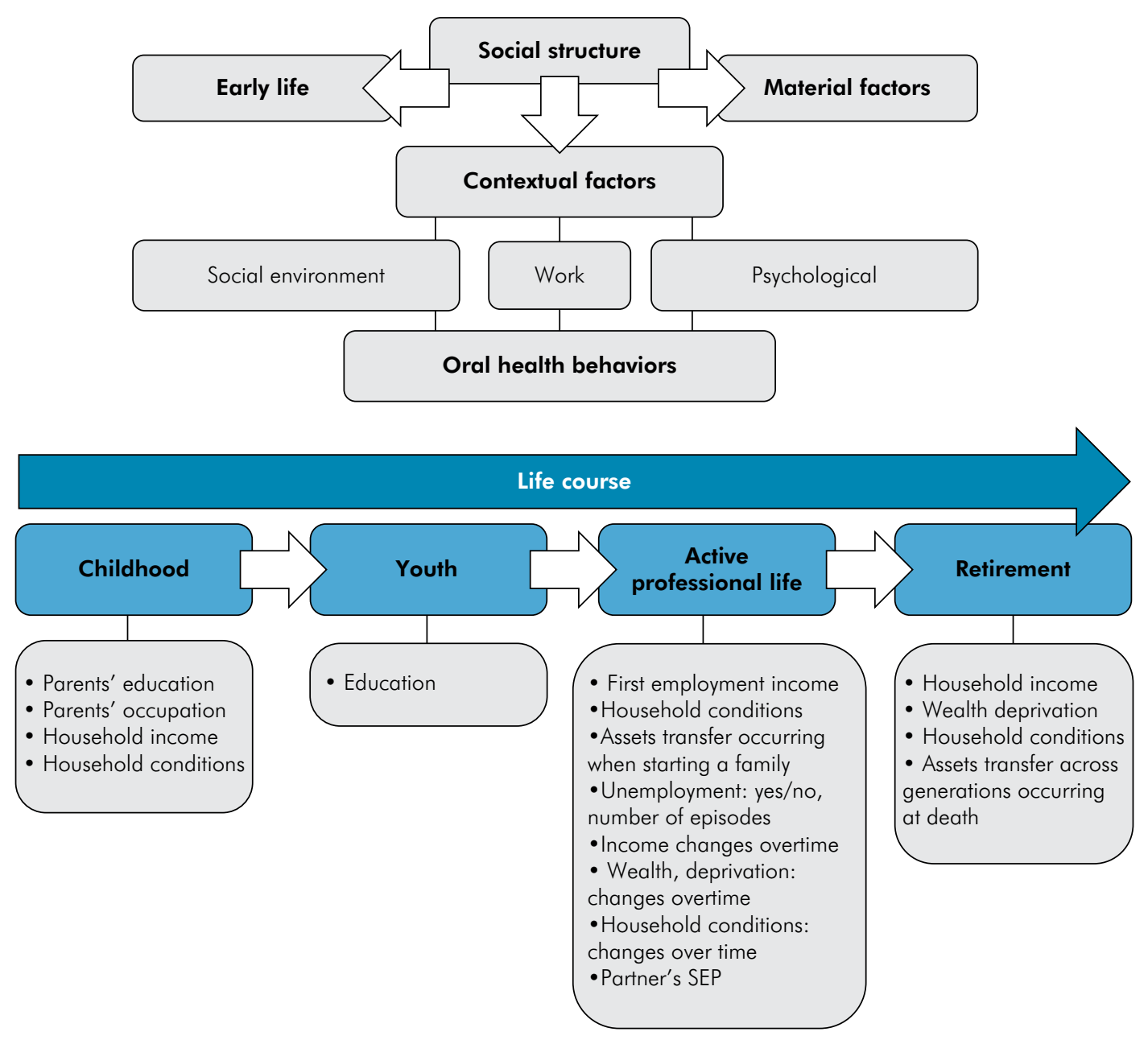

Figure 3. Indicators measuring life course socioeconomic position on an individual basis. Adapted from Galobardes et al. ${ }^{21}$ and Thomson et al. ${ }^{19}$

Education

Impacts on each factor in each context at each level

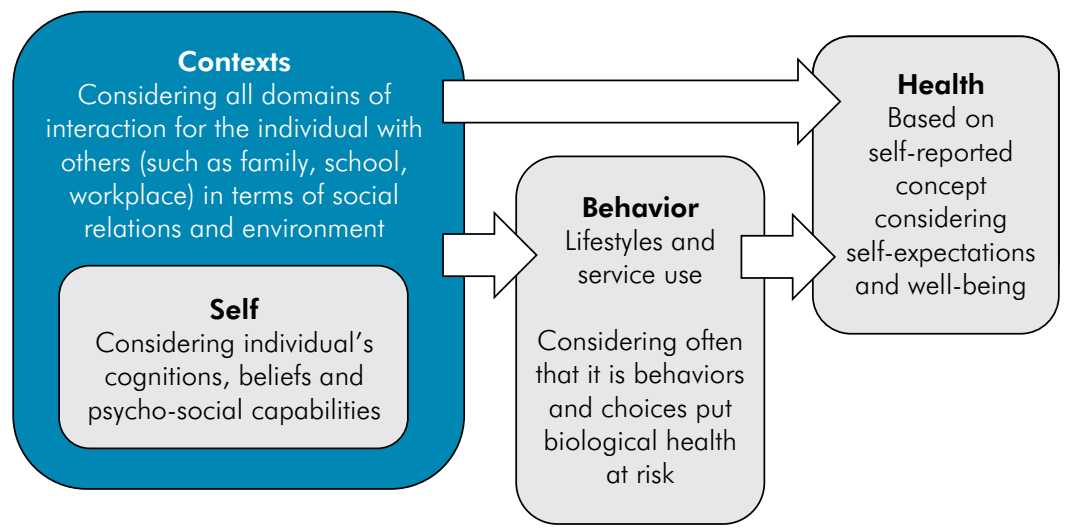

Figure 4. How education affects an individual's life in all contexts and levels, including lifestyles that can positively or negatively influence health. Adapted from Feinstein et al. ${ }^{23}$ 
improving health literacy reduces healthcare costs, increases health knowledge, decreases hospital stay, and reduces the use of healthcare services. It is also relevant to considerer antecedents like demographic, psychosocial, and cultural factors, including education. ${ }^{26}$

A theoretical framework has been proposed to explain the association between health literacy and periodontal outcomes (Figure 5). ${ }^{27}$ A systematic review found an inverse relationship between number of teeth and clinical attachment loss and oral health literacy. However, the association between the latter and deep periodontal pockets, bleeding on probing, severity of periodontal disease, and dental plaque was unclear. ${ }^{28}$

Interestingly, the association between oral health literacy (OHL) and periodontal parameters depends on the OHL measurement tools used. ${ }^{27}$ Additionally, the association between $\mathrm{OHL}$ and periodontal parameters has been supported by few studies that recruited patients seeking dental care. Thus, further studies involving larger and representative samples of the general population are needed.

\section{Behavioral change interventions}

The effectiveness of periodontal disease prevention largely depends on modifications of behavior, knowledge, health literacy, patient empowerment, motivation, and compliance. Overall, there is inadequate knowledge, attitudes, and practices related to periodontal disease prevention. Therefore, behavioral factors are key concerns to prevent periodontal diseases. ${ }^{18}$
Promoting behavioral and lifestyle changes to reduce risk factors and optimize long-term adherence to professional advice is essential in the prevention and management of chronic conditions, including periodontal diseases..$^{29}$ Previous systematic reviews drew cautious conclusions that interventions based on psychological theories aiming to change behaviors provided better results than nontheory-based or simple educational interventions. ${ }^{29,30}$ There is some evidence that oral hygiene in patients with periodontal diseases may be improved by oral health education based on behavioral interventions and motivational interviewing. ${ }^{18}$

There are different interventions based on different psychological theories and behavioral models. ${ }^{18,31}$ These interventions are based on the assumption that many psychological factors (e.g. oral health beliefs, locus of control, sense of coherence, self-efficacy, dental anxiety) are related to oral health behaviors (e.g. compliance with and adherence to professional advice and regular maintenance dental visits). ${ }^{31}$ However, the lack of a single unifying psychological framework related to behavioral changes limits possible conclusions on the effectiveness of these interventions, as well as recommendations for their use at individual or population levels. ${ }^{32}$

A comprehensive framework focusing on the nature of the intervention and behavioral constructs necessary to encourage behavioral changes suggests that the interventions should target one of the following elements: a) capability (physical and psychological skills to perform the behavior); $b$ )

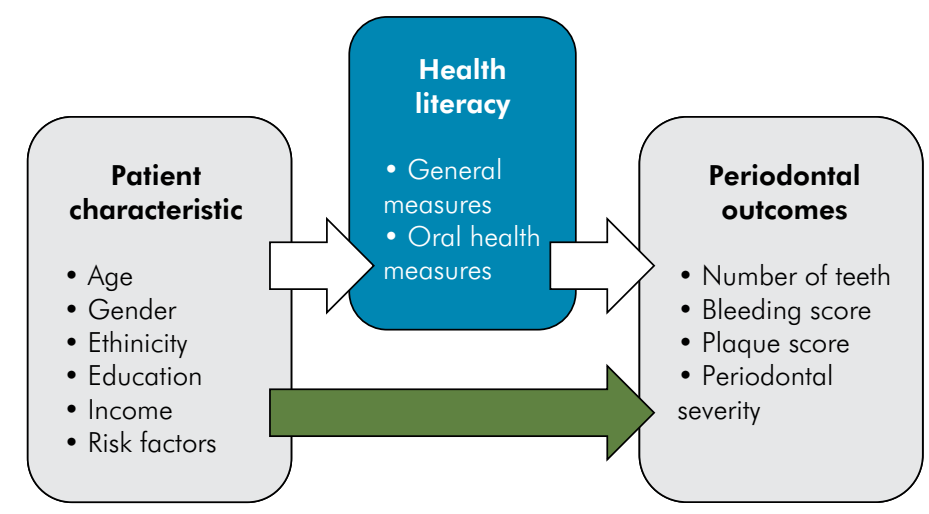

Figure 5. Relation between health literacy and periodontal outcomes. Adapted from Holtzman et al. ${ }^{27}$ 
opportunity (physical and social environment supporting the new behavior; c) motivation (conscious and automatic processes underlying the occurrence of the behavior) (Figure 6). ${ }^{33}$ Based on the analysis of which element is currently deficient, this model can provide a "behavioral diagnosis" and identify the nature of the intervention needed at the individual level (e.g. education, persuasion, training, coercion, and modeling) and at the population level, within a broader social and environmental setting (e.g. service provision, social planning, health strategies, fiscal measures, and legislation). ${ }^{32}$

Approaches based on a three-component intervention: a) goal setting; b) planning the behavior change; c) monitoring the behavior (either by the individuals themselves or by a healthcare professional providing feedback) are effective in improving personal oral hygiene in periodontal patients. ${ }^{32,34}$ Evidence also suggests that this approach can be used in primary care settings. ${ }^{35}$

The commonly used information-giving approach to behavioral change in dental practice presents little effect on sustainable gain of dental knowledge or self-efficacy beliefs. Another approach to behavioral change and motivation improvement is motivational interviewing. It is a collaborative goal-oriented communication method focused on the language of change, aiming to strengthen personal motivation and commitment to a specific goal. ${ }^{31,36}$ A systematic overview on the effectiveness of motivational interviewing about different health problems and diseases demonstrated effective results in stopping or preventing unhealthy lifestyle behaviors, such as smoking, alcohol consumption, and substance abuse. ${ }^{36}$ Interventions based on motivational interviewing in periodontal clinical settings provided less consistent effectiveness. This may result from either poor operationalization of the intervention by dental professionals or genuine failure of the approach. ${ }^{32}$

Current evidence indicates that behavioral interventions based on cognitive constructs and motivational interviewing principles have a positive impact on an individual's behavioral changes that may result in additional benefits to the standard periodontal treatment and oral hygiene reinforcement. However, no significant clinical benefits for the reduction of dental plaque and gingival bleeding over time have been observed. ${ }^{31}$

The use of mobile technologies to orientate and improve health outcomes is known as mobile health (mHealth) ${ }^{37}$ It is described as individual and public health practices supported by mobile devices that

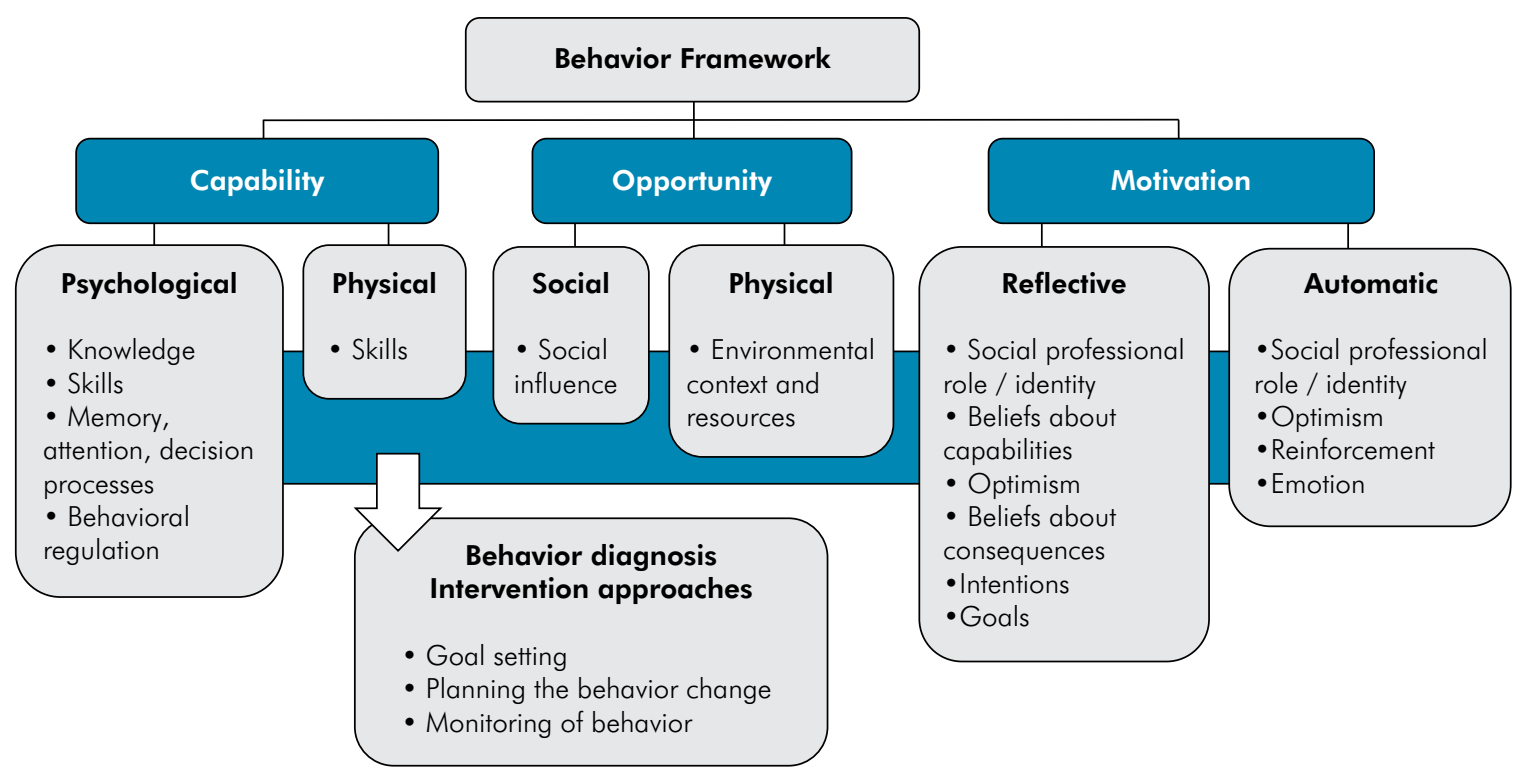

Figure 6. Behavior constructs framework based on Behavioral models for periodontal health and disease. Adapted from Newton and Asimakopoulou. ${ }^{32}$ 
can facilitate communication and patient education, management, and monitoring. Therefore, they can improve motivation, compliance, and health self-care knowledge. ${ }^{37,38}$ Compared to traditional approaches, interactive multimedia and visually attractive materials are more appropriate to communicate with and motivate patients. Text messages can provide warnings, reminders, reinforcements, and feedback, which are important factors to promote behavioral changes and health literacy. ${ }^{38}$

Findings from a recent meta-analysis indicate improved oral hygiene and significant reduction in dental plaque and gingival bleeding among individuals receiving mHealth strategies, with better results among adolescents. Text messages and mobile applications can enhance daily self-performed oral hygiene and increase oral health literacy. Notwithstanding the promising benefits of $\mathrm{mHealth}$ in self-care, the effectiveness of mHealth is still considered modest. ${ }^{38}$

Evidence of the effectiveness of other communication methods, such as self-inspection and videotapes, to improve oral hygiene is also unclear. Overall, possible advantages of one psychological intervention over another are yet difficult to establish. ${ }^{31}$

\section{Conclusions}

a. Patient-centered preventive models are more effective than professional-centered models in the management of periodontal diseases;

b. There is enough evidence supporting the mechanical and chemical biofilm control as part of preventive measures in periodontal diseases;

c. Effective periodontal care requires comprehensive preventive approaches both at individual and population levels, including the common risk factor approach;

d. The prevention of periodontal diseases requires the improvement of knowledge, better health literacy, patient empowerment, motivation, and compliance.

\section{Acknowledgments}

The authors are grateful to Dr. Marcelo W. B. Araujo, who shared new perspectives and insights that improved the manuscript.

\section{References}

1. Tonetti MS, Greenwell H, Kornman KS. Staging and grading of periodontitis: framework and proposal of a new classification and case definition. J Periodontol. 2018 Jun;89 Suppl 1:S159-72. https://doi.org/10.1002/JPER.18-0006

2. Baehni PC. Translating science into action: prevention of periodontal disease at patient level. Periodontol 2000. 2012 Oct;60(1):162-72. https://doi.org/10.1111/j.1600-0757.2011.00428.x

3. Bouchard P, Carra MC, Boillot A, Mora F, Rangé H. Risk factors in periodontology: a conceptual framework. J Clin Periodontol. 2017 Feb;44(2):125-31. https://doi.org/10.1111/icpe.12650

4. Rose G. The strategy of preventive medicine. Oxford: OUP; 1993.

5. Murakami S, Mealey BL, Mariotti A, Chapple IL. Dental plaque-induced gingival conditions. J Periodontol. 2018 Jun;89 Suppl 1:S17-27. https://doi.org/10.1002/JPER.17-0095

6. Lang NP, Schätzle MA, Löe H. Gingivitis as a risk factor in periodontal disease. J Clin Periodontol. 2009 Jul;36 Suppl 10:3-8. https://doi.org/10.1111/j.1600-051X.2009.01415.x

7. Van der Weijden FA, Slot DE. Efficacy of homecare regimens for mechanical plaque removal in managing gingivitis a meta review. J Clin Periodontol. 2015 Apr;42 Suppl 16:S77-91. https://doi.org/10.1111/icpe.12359

8. Sälzer S, Slot DE, Van der Weijden FA, Dörfer CE. Efficacy of inter-dental mechanical plaque control in managing gingivitis: a meta-review. J Clin Periodontol. 2015 Apr;42 Suppl 16:S92-105. https://doi.org/10.1111/icpe.12363

9. Needleman I, Nibali L, Di lorio A. Professional mechanical plaque removal for prevention of periodontal diseases in adults: systematic review update. J Clin Periodontol. 2015 Apr;42 Suppl 16:S12-35. https://doi.org/10.1111/icpe.12341

10. Serrano J, Escribano M, Roldán S, Martín C, Herrera D. Efficacy of adjunctive anti-plaque chemical agents in managing gingivitis: a systematic review and meta-analysis. J Clin Periodontol. 2015 Apr;42 Suppl 16:S106-38. https://doi.org/10.1111/icpe.12331

11. Figuero E, Herrera D, Tobías A, Serrano J, Roldán S, Escribano M, et al. Efficacy of adjunctive anti-plaque chemical agents in managing gingivitis: a systematic review and network meta-analyses. J Clin Periodontol. 2019 Jul;46(7):723-39. https://doi.org/10.1111/icpe.13127 
- Periodontal diseases: is it possible to prevent them? A populational and individual approach

12. Van Leeuwen MP, Slot DE, Van der Weijden GA. Essential oils compared to chlorhexidine with respect to plaque and parameters of gingival inflammation: a systematic review. J Periodontol. 2011 Feb;82(2):174-94. https://doi.org/10.1902/jop.2010.100266

13. Araujo MW, Charles CA, Weinstein RB, McGuire JA, Parikh-Das AM, Du Q, et al. Meta-analysis of the effect of an essential oil-containing mouthrinse on gingivitis and plaque. J Am Dent Assoc. 2015 Aug;146(8):610-22. https://doi.org/10.1016/i.adaj.2015.02.011

14. Quirynen M, Soers C, Desnyder M, Dekeyser C, Pauwels M, Steenberghe D. A 0.05\% cetyl pyridinium chloride/0.05\% chlorhexidine mouth rinse during maintenance phase after initial periodontal therapy. J Clin Periodontol. 2005 Apr;32(4):390-400. https://doi.org/10.1111/j.1600-051X.2005.00685.x

15. Sheiham A, Watt RG. The common risk factor approach: a rational basis for promoting oral health. Community Dent Oral Epidemiol. 2000 Dec;28(6):399-406. https://doi.org/10.1034/j.1600-0528.2000.028006399.x

16. World Health Organization. Strategies and approaches in oral disease prevention and health promotion. Geneva: World Health Organization; 2020 [cited 2020 Sept 16]. Available from: www.who.int/oral_health/strategies/cont/en/

17. Watt RG, Petersen PE. Periodontal health through public health: the case for oral health promotion. Periodontol 2000. 2012 Oct;60(1):147-55. https://doi.org/10.1111/j.1600-0757.2011.00426.x

18. Duque AD, Malheiros Z, Stewart B, Romanelli HJ. Strategies for the prevention of periodontal disease and its impact on general health in Latin America. Section III: Prevention. Braz Oral Res. 2020 Apr 9;34(supp 1):e025. https://doi.org/10.1590/1807-3107bor-2020.vol34.0025

19. Thomson WM, Sheiham A, Spencer AJ. Sociobehavioral aspects of periodontal disease. Periodontol 2000. 2012 Oct;60(1):54-63. https://doi.org/10.1111/j.1600-0757.2011.00405.x

20. Schuch HS, Peres KG, Singh A, Peres MA, Do LG. Socioeconomic position during life and periodontitis in adulthood: a systematic review. Community Dent Oral Epidemiol. 2017 Jun;45(3):201-8. https://doi.org/10.1111/cdoe.12278

21. Galobardes B, Shaw M, Lawlor DA, Lynch JW, Davey Smith G. Indicators of socioeconomic position (part 1). J Epidemiol Community Health. 2006 Jan;60(1):7-12. https://doi.org/10.1136/jech.2004.023531

22. Borrell LN, Crawford ND. Socioeconomic position indicators and periodontitis: examining the evidence. Periodontol 2000. 2012 Feb;58(1):69-83. https://doi.org/10.1111/j.1600-0757.2011.00416.x

23. Feinstein L, Sabates R, Anderson TM, Sorhaindo A, Hammond C. What are the effects of education on health? Measuring the effects of education on health and civic/social engagement: proceedings of the Kopenhagen symposium. Paris: OECD; 2006 [cited 2020 Nov 23]. Available from: http://www.oecd.org/education/innovation-education/37425753.pdf

24. Wittink H, Oosterhaven J. Patient education and health literacy. Musculoskelet Sci Pract. 2018 Dec;38:120-7. https://doi.org/10.1016/i.msksp.2018.06.004

25. Nutbeam D. Health literacy as a public goal: a challenge for contemporary health education and communication strategies into the $21 \mathrm{st}$ century. Health Promot Int. 2000 Sep;15(3):259-67. https://doi.org/10.1093/heapro/15.3.259

26. Sørensen K, Van den Broucke S, Fullam J, Doyle G, Pelikan J, Slonska Z. Health literacy and public health: a systematic review and integration of definitions and models. BMC Public Health. 2012 Jan;12(1):80. https://doi.org/10.1186/1471-2458-12-80

27. Holtzman JS, Atchison KA, Macek MD, Markovic D. Oral Health Literacy and Measures of Periodontal Disease. J Periodontol. 2017 Jan;88(1):78-88. https://doi.org/10.1902/jop.2016.160203

28. Firmino RT, Ferreira FM, Paiva SM, Granville-Garcia AF, Fraiz FC, Martins CC. Oral health literacy and associated oral conditions: a systematic review. J Am Dent Assoc. 2017 Aug;148(8):604-13. https://doi.org/10.1016/i.adaj.2017.04.012

29. Renz A, Ide M, Newton T, Robinson PG, Smith D. Psychological interventions to improve adherence to oral hygiene instructions in adults with periodontal diseases. Cochrane Database Syst Rev. 2007 Apr;(2):CD005097. https://doi.org/10.1002/14651858.CD005097.pub2

30. Werner H, Hakeberg M, Dahlström L, Eriksson M, Siögren P, Strandell A, et al. Psychological interventions for poor oral health: a systematic review. J Dent Res. 2016 May;95(5):506-14. https://doi.org/10.1177/0022034516628506

31. Carra MC, Detzen L, Kitzmann J, Woelber JP, Ramseier CA, Bouchard P. Promoting behavioural changes to improve oral hygiene in patients with periodontal diseases: a systematic review. J Clin Periodontol. 2020 Jul;47(S22 Suppl 22):72-89. https://doi.org/10.1111/jcpe.13234

32. Newton JT, Asimakopoulou K. Behavioral models for periodontal health and disease. Periodontol 2000. 2018 Oct;78(1):201-11. https://doi.org/10.1111/prd.12236

33. Michie S, van Stralen MM, West R. The behaviour change wheel: a new method for characterising and designing behaviour change interventions. Implement Sci. 2011 Apr;6(1):42. https://doi.org/10.1186/1748-5908-6-42

34. Newton JT, Asimakopoulou K. Managing oral hygiene as a risk factor for periodontal disease: a systematic review of psychological approaches to behaviour change for improved plaque control in periodontal management. J Clin Periodontol. 2015 Apr;42 Suppl 16:S36-46. https://doi.org/10.1111/icpe.12356

35. Suresh R, Jones KC, Newton JT, Asimakopoulou K. An exploratory study into whether self-monitoring improves adherence to daily flossing among dental patients. J Public Health Dent. 2012;72(1):1-7. https://doi.org/10.1111/j.1752-7325.2011.00274.x 
36. Frost $\mathrm{H}$, Campbell $\mathrm{P}$, Maxwell M, $\mathrm{O}^{\prime}$ Carroll RE, Dombrowski SU, Williams $B$, et al. Effectiveness of Motivational interviewing on adult behaviour change in health and social care settings: a systematic review of reviews. PLoS One. 2018 Oct;13(10):e0204890. https://doi.org/10.1371/journal.pone.0204890

37. World Health Organization. mHealth: New horizons for health through mobile Technologies: second global survey on eHealth. Geneva: Global Observatory for eHealth Series; 2011.

38. Toniazzo MP, Nodari D, Muniz FW, Weidlich P. Effect of mHealth in improving oral hygiene: A systematic review with meta-analysis. J Clin Periodontol. 2019 Mar;46(3):297-309. https://doi.org/10.1111/icpe.13083 